\title{
Mucoadhesive Micro and Nanoparticles for Oral Controlled Drug Delivery System for Prolongation of Gastric Residence and Its Application
}

\section{Suryakanta Swain*}

Department of Pharmaceutics, Roland Institute of Pharmaceutical Sciences, India

\section{Editorial}

The healthcare system has been highly impacted by drug delivery systems that deals with controlling release rate or leads drugs to a predetermined body site. Last two decades have witnessed phenomenal growth in sector of polymer and material science redefining the development of novel drug delivery systems.

Coming to mucoadhesion concept, it is basically the formation of hydrogen bonds and ionic interactions that is precipitated by physical entanglements of mucus gel layer and polymers. New generation mucoadhesive polymers play a pivotal role in enhancing the residence time of drug delivery systems on the intestinal mucosa to maximize the therapeutic effect through prolong the sustainability of the drug.

Carrier technology another vibrate facet of NDDS is an innovative approach. Its prime concern is the delivery of the drug by coupling it to a carrier particle and thereby alters the release and absorption characteristics of the drug. Microspheres, nanoparticles, nanocapsules and liposome, are various popular current forms of carrier technology.

Furthermore, drug delivery systems can be localized on a certain surface area for the purpose of local therapy or of drug liberation at the 'absorption window' representing the GI-segment, where drug absorption takes place. Absorption of riboflavin, which has its 'absorption window' in the stomach and upper segment of the small intestine, could be strongly improved in human volunteers by oral administration of mucoadhesive microspheres versus non-adhesive microspheres. The potential of thiomers to enhance and prolong the mucosal uptake of poorly absorbed drugs could meanwhile be shown for various challenging therapeutic agents such as hydrophilic macromolecular drugs in various in vivo studies. The oral administration of low molecular weight being embedded in thiolated polycarbophil resulted in a significantly increased absorption of heparin compared to control tablets comprising unmodified polycarbophil or to an orally given aqueous heparin solution.

The present editorial explores mucoadhesive oral drug delivery system based on thermally-hydrocarbonized porous silicon (THCPSi). It is further associated with a self-assembled amphiphilic protein. Class II hydrophobin (HFBII) from Trichoderma reesei is used as a coating of the nanoparticles. It is found that the outcome is non-cytotoxic. It is strongly recommended that it may be used in AGS cells and biodistribution study in rats after oral administration.

Another approach for delivery of drug by gastroretentivemucoadhesive system by using PLGA/chitosan microparticles is worth consideration. It has the potential to be used as controlled release delivery system for prolongation of gastric residence. Supercritical $\mathrm{CO}_{2}$ by the Particle Gas Saturated System (PGSS) or CriticalMix(TM) technique is used for it.

Treatment of bladder-related diseases is tackled with the help of an epoch making approach i.e. nanomedical tool based on thiolated chitosan and biodegradable PLGA. Similarly hepatitis B is treated with the help of lectin anchored PLGA nanoparticles and M-cell with a view to immunize oral mucosa. Intranasally administered surface-modified PLGA microspheres are also in the race to provide strong systemic and mucosal immune responses against recombinant hepatitis B antigen.

On behalf of the editor and editorial board for the upcoming issue, I would like to thank the OMICS publishing Group 5716 Corsa Ave., Suite 110 Westlake, Los Angeles, CA 91362-7354, USA, for giving me this opportunity to write an invited editorial article.

Citation: Swain S (2012) Mucoadhesive Micro and Nanoparticles for Ora Controlled Drug Delivery System for Prolongation of Gastric Residence and Its Application. Pharmaceut Reg Affairs 1:e115. doi:10.4172/2167-7689.1000e115

Copyright: @ 2012 Swain S. This is an open-access article distributed under the terms of the Creative Commons Attribution License, which permits unrestricted use, distribution, and reproduction in any medium, provided the original author and source are credited. 